\title{
Somatic Mutations in Benign Breast Disease Tissues and Association With Breast Cancer Risk
}

Stacey Winham ( $\nabla$ winham.stacey@mayo.edu )

Mayo Clinic Rochester https://orcid.org/0000-0002-8492-9102

Chen Wang

Mayo Clinic

Ethan P. Heinzen

Mayo Clinic

Aditya Bhagwate

Mayo Clinic

Yuanhang Liu

Mayo Clinic

Samantha J. McDonough

Mayo Clinic

Melody L. Stallings-Mann

Mayo Clinic

Marlene H. Frost

Mayo Clinic

Robert A. Vierkant

Mayo Clinic

Lori A. Denison

Mayo Clinic

Jodi M. Carter

Mayo Clinic

Mark E. Sherman

Mayo Clinic

Derek C. Radisky

Mayo Clinic

Amy C. Degnim

Mayo Clinic

Julie M. Cunningham

Mayo Clinic

Research article 
Keywords: Breast Cancer risk, Benign Breast Disease, Somatic mutations, Mutation burden, CD45 expression Posted Date: February 26th, 2021

DOI: https://doi.org/10.21203/rs.3.rs-257991/v1

License: () (i) This work is licensed under a Creative Commons Attribution 4.0 International License. Read Full License

Version of Record: A version of this preprint was published at BMC Medical Genomics on July 14th, 2021. See the published version at https://doi.org/10.1186/s12920-021-01032-8. 


\section{Abstract}

Background: Benign breast disease (BBD) is a risk factor for breast cancer (BC); however, little is known about the genetic alterations present at the time of BBD diagnosis, and how these relate to risk of incident BC.

Methods: A subset of a long-term BBD cohort was selected to examine DNA variation across three BBD groups (42 future estrogen receptor-positive (ER+) BC, 36 future estrogen receptor-negative (ER-) BC and 42 controls cancer-free for at least 16 years post-BBD). DNA extracted from archival formalin fixed, paraffinembedded (FFPE) tissue blocks was tested and analyzed for presence of DNA alterations using a targeted panel of $93 \mathrm{BC}$-associated genes. To address artifacts frequently observed in FFPE tissues (e.g., C>T changes), we applied three filtering strategies based on alternative allele frequencies and nucleotide substitution context. Gene-level associations were performed using two burden tests, SKAT-O and logistic regression, and adjusted for clinical and technical covariates including epithelial percentage, histologic impression, patient age and year of BBD biopsy.

Results: After filtering, the variant frequency of SNPs in our samples was highly consistent with population allele frequencies reported in $1 \mathrm{KG} / \mathrm{ExAC}(0.986, \mathrm{p}<1 \mathrm{e}-16)$. Ten genes were found to be associated with later cancer status by four of 12 association methods, with nominal p-values <0.05: MED12, MSH2, BRIP1, PMS1, GATA3, MUC16, FAM175A, EXT2, MLH1 and TGFB1. Additionally, analysis revealed 11 and 19 gene-level associations with $\mathrm{p}$-values $<0.05$ and $\mathrm{OR}<1$ for all cancer cases and ER-cases, respectively. Comparing between the three case groups, BBD ER+ cases were closer to controls in mutation profile, while BBD ERcases were distinct. Notably, the variant burden was significantly higher in controls than in either ER+ or ERcases. CD45 expression was associated with mutational burden $(p=0.003)$.

Conclusions: Somatic mutations were more frequent in benign breast tissue from women who did not develop cancer, opening questions of clonal diversity or immune-mediated restraint on future cancer development. CD45 expression was positively associated with mutational burden, most strongly in controls. Further studies in both normal and premalignant tissues are needed to better understand the role of somatic gene mutations and their contribution to future cancer development.

\section{Introduction}

Breast cancer $(\mathrm{BC})$ is a leading cause of cancer in women, and is believed to result from the progressive accumulation of genetic and epigenetic alterations, where genomic analyses have revealed both inherited predisposition and common genetic variation playing a role in susceptibility [1-3]. However, the majority of women with $\mathrm{BC}$ have no major germline mutations and thus develop sporadic cancers, raising questions. Early detection is critical to detect and prevent cancer-related death, as early-stage BC has $99 \%$ five-year survival rate, compared to $27 \%$ for advanced stage cancers [4]. Identification of biomarkers that predict progression in cancer precursors could enable improved management, where high-risk women are offered closer surveillance and preventative treatments, whereas low-risk women may be screened less frequently. Benign breast disease (BBD), which includes non-proliferative, proliferative and proliferative lesions with 
atypia, is viewed as a nonobligate precursor stage in the development of $\mathrm{BC}$ [5], and is associated with an increased risk of invasive BC, particularly in those with proliferative or atypical lesions [6-8].

As the majority of women with $\mathrm{BC}$ have no major germline mutations and thus develop sporadic cancer, the platform of benign breast disease offers a window in early carcinogenetic events. Questions remain regarding the processes that drive deoxyribonucleic acid (DNA) mutations in breast tissue for patients with sporadic $\mathrm{BC}$, and which mutations are associated with the earliest stages of $\mathrm{BC}$ development. In particular, atypical hyperplasia has been reported to share genomic changes with common sporadic BC, including structural genomic changes such as aneuploidy, loss of heterozygosity, and large-scale amplifications and deletions $[9,10]$. In addition to structural alterations, analysis of DNA mutations present in BBD biopsies, which contain both the BBD lesion and the surrounding tissue bed, has the potential to define the processes that drive the development of cancer-associated DNA mutations, as well as which of those mutations are most critical for cancer development.[9, 10]

To date, few DNA sequencing studies have examined mutational status in BBD tissues. Rohan et al [11] sequenced 218 BBD cases that subsequently developed invasive BC and matched cancer free BBD controls using a targeted capture-based panel and reported no significant mutation burden differences. Adjacent, non-malignant tissue was used as a surrogate for germline variants which were excluded. Soysal et al [12] used a targeted amplicon-based sequencing panel to profile 17 cases of invasive $\mathrm{BC}$ with a previous diagnosis of fibrocystic disease, all with matching normal tissue. They reported that no significant mutations in hotspot residues were seen in either tumor or benign disease. A recent report by Zeng et al [13] details whole exome sequencing on 135 BBD cases that subsequently developed cancer and 69 cancer free controls, using a "pool of normals" and a neural network to predict somatic variants in the unrelated BBD samples. While finding no significant difference in the number of mutations between cases and controls, when filtering to variants with variant allele frequency $(\mathrm{VAF})>25 \%$, non-silent mutation differences were observed between cases and controls for some but not all genes detected at lower VAF.

The Mayo Clinic BBD cohort was first described in 2005 [5], and includes more than 13,258 BBD cases with a median follow-up of 13 years of clinical data [14]. To identify underlying genetic aberrations in BBD cases associated with future BC occurrences, we designed a DNA sequencing study of 120 patients with formalinfixed paraffin-embedded (FFPE) BBD tissues. This study focused on three groups, those patients who remained cancer-free after at least 16 years post-BBD (controls), and those developing estrogen receptor positive (ER+) or estrogen receptor negative (ER-) invasive BC cancers within 16 years. Analysis focused on comparison of controls to the cancer groups, and between ER+ and ER- cancers.

\section{Methods}

\section{Cohort constructions}

The Mayo Clinic Benign Breast Disease (BBD) Cohort includes 13,455 women, ages 18-85 who underwent benign biopsies at Mayo Clinic between 1967 and 2001. Women who had been diagnosed with invasive or in situ $\mathrm{BC}$ before or within six months of biopsy or have undergone risk-reducing mastectomy or breast 
reduction surgery prior to biopsy were excluded. Among this cohort, a frequency-matched (by age and year of biopsy) case-control sample was selected, where cases were defined as those women with BBD who subsequently went on to develop either ER + or ER- BC within 16 years, and controls were defined as women with BBD who had not developed BC after at least 16 years of follow-up. Index benign biopsies were screened from women with ER- or ER + BC and corresponding controls, matched on age at biopsy, year of biopsy, and length of follow-up time/time to BC diagnosis. After determining tissue block availability, adequate DNA amount and quality, and adequate sequencing quality, our final sample set for association analysis included 42 ER + cases, 36 ER- cases, and 42 controls diagnosed between 1969 and 2001.

\section{DNA extraction \& Sequencing}

DNA extraction and sequencing were performed as previously reported [15]. In brief, DNA was extracted from ten micrometer sections of FFPE or fresh frozen tissue using the GeneRead DNA FFPE kit (Qiagen, Germantown, MD, US). After extraction, DNA was quantified using Qubit ${ }^{\text {TM }}$ dsDNA BR Assay (ThermoFisher Scientific, Waltham, MA, USA) while quality was assessed using the Advanced Analytical Fragment Analyzer $^{\text {TM }}$ High Sensitivity Large Fragment Analysis kit which calculates fragment length and degradation.

The QIAseq Human Breast Cancer Targeted Panel, which targets 93 genes relevant in BC, was used to create libraries using 20-40 ng of DNA as previously reported [15], following Qiagen guidelines for FFPE DNA. Libraries were quantified and sequenced on an Illumina ${ }^{\circledR}$ HiSeq 4000 (Illumina, San Diego, CA, USA) paired end 150-bp.

To facilitate quality control and to provide confidence in results derived from archival FFPE tissue, a set of technical controls samples was used, and included four fresh snap-frozen benign breast samples (with pathologic assessment of cryoH\&E sections) and paired FFPE tissues from reduction mammoplasties, one CEPH control NA12891 (Coriell Institute for Medical Research, Camden, NJ, USA), one FFPE BBD sample not in the sample set, and a positive control sample from formalin fixed cell lines with 11 mutations at varying allelic frequencies (Horizon Diagnostics LLC, Columbus, GA, USA). Variants were located within the BRAF, CKIT, EGFR, KRAS, NRAS and PIK3CA genes.

\section{DNA-seq Alignment and Variant calling}

The Qiagen Data Portal was used for primary sequencing analysis of the samples [21]. The analysis steps included adapter trimming, coupling molecular tag (MT) sequence to the read IDs, alignment to the reference genome (GRCh37 build), and subsequent variant calling using smCounter, a molecular tag-aware variant calling algorithm. smCounter uses a Bayesian probabilistic model to identify variants and infer genotypes and can detect low frequency variants with high sensitivity [22]. As each unique DNA fragment generated from the experiment should have a unique molecular tag attached, sequenced reads with identical molecular tags were identified as PCR duplicates. During analysis, such reads arising from PCR duplicates were collapsed to create a consensus read sequence. In order to reflect molecular diversities of each sample, a socalled molecular diversity score was defined as proportion of molecular-tag coverage versus raw sequencing coverage (100 x MT-coverage/Raw-coverage). For variant calling, the consensus sequence was compared to the reference genome and the smCounter algorithm calculates a prediction index of the alleles observed at the barcode level for every target position and a variant is called if an allele shows a higher prediction index 
compared to a preselected prediction index threshold. This threshold is based on the demonstration by smCounter that 8 reads per barcode is sufficient for variant analysis as described in [22]. The output from the variant calling step was obtained in the standard VCF. After variant-calling, initial variant filtering was done to exclude likely false calls due to technical factors, such as presence in low complexity regions, shallow molecular tag coverage, strand bias, and/or low base quality. Only variants passing filter recommendations by the QIAGEN@ (QIAGEN, Germantown, MD, USA) bioinformatics pipeline were used for subsequent analysis.

\section{Sample QC and acceptance criteria}

Samples with an average unique molecular tag (UMT) coverage of $<20 x$ and with genotyping call rate of SNPs $<80 \%$ were excluded. For each sample, variants were called as genotypes based on bins of allele frequencies of 0-0.2 (rare homozygote), 0.4-0.6 (heterozygote), and 0.8-1.0 (common homozygote), and the genotyping call rate was defined as the proportion of SNPs for which the genotype was called. The distribution of genotyping call-rate versus the mean UMT coverage is shown in Additional File 1.

Sample identity was examined using Spearman correlation of the minor allele frequency of known SNPS across all samples to identify those emanating from the same individual. For quality control, 24 samples with two to 10 replicates were profiled independently, with a total number of 119 replicate-pairs. After strict variant filtering, correlation values of replicates were completely separable from unrelated samples; replicate samples had correlation close to 1.0 (all $>0.85$ ), whereas unrelated samples had correlation centered around 0.6 (all < 0.85) [See Additional File 2. Metadata on the final sample set are presented in Additional File 3.]

\section{Additional Variant Filtering and population allele frequency concordance}

As FFPE samples are known to be prone to variant artifacts, additional filtering was required before further analysis. Utilizing paired FFPE and fresh frozen samples we studied previously with identical methods[15], false discovery rate $(F D R)$ of variant-calling was approximated for seven mutation types $(C>A, C>G, C>T$, $C>T$ at $C p G, T>A, T>C$ and $T>G$ ) [See Additional File 4]. The empirical relationships between alternate allele frequency (AAF) and FDR were utilized to determine further filtering strategies; variants with AAF smaller than a certain cutoff value ( 0.05 or 0.1$)$ based on stringency were removed.

After additional AAF-based filtering, variant frequencies in our cohort were compared with population allele frequencies to ensure that additional filtering strategies did not skew allele distribution of study samples. In particular, all the detected variants were annotated with population allele frequencies observed in 1000 genome project \& ExAC project based on internally developed bioR annotation software [23], and were compared between the overlapping variants.

\section{Mutational Signature Analysis}

Using the filtered variant sets (see above), a set of custom Perl scripts was used to generate a mutation frequency table of all SNVs across each sample to assess the of mutation types being reported. This mutation frequency table was subsequently used to generate plots of mutation signatures for the variants 
using Perl and R scripts. Mutational spectrums and de-novo mutational signatures were identified using MutationalPatterns package (version 1.2.1) [24]. De-novo signatures were extracted based on a non-negative matrix factorization (NMF) algorithm. Through consensus clustering, four stable de-novo mutational signatures were identified and compared with COSMIC mutational signatures v2 $(n=30)$ based on cosine similarity [Additional File 5] [25]. Signature A (QGR signature) matches the patterns identified in samples processed with the FFPE DNA protocol used, the QIAGEN GeneRead DNA FFPE Kit ("QGR") [15]. Signature B (FFPE signature) matches patterns observed in FFPE samples but not in fresh-frozen samples. Signature $C$ (Block year signature) was highly associated with the year of FFPE block creation. Signature D (Residual signature) had no clear correspondence with previously found mutational signatures. The estimated signatures are shown in Additional File 6.

\section{Gene-level Association methods}

To prioritize variants for association analyses, those meeting any of the following criteria were removed: 1 . Observed in common populations with minor allele frequency (MAF) $>0.5 \%$, according to Genome Aggregation Database (gnomAD) and Trans-Omics for Precision Medicine (TOPMED) studies[26, 27]. 2. Defined as low functional impact by the CAVA bioinformatics annotation tool [28]. In the CAVA annotation process, medium and high impact variants were defined as essential splice bases, stop gain, frameshift, nonsynonymous variant, inframe indels, start codon, stop loss, and/or exon end (alters first or last three bases of exon).

We further defined three sets of variants based on liberal, classical or strict filtering of AAF. For the liberal set, variants with $\mathrm{AAF}<0.05$ were removed. For the classical set, $\mathrm{C}>\mathrm{T}$ mutations (with the exception of SNPS annotated with an $\mathrm{rSID}$ ) with $\mathrm{AAF}<0.1$ were removed, while other types of mutations with $\mathrm{AAF}<0.05$ were removed. For the strict set, all variants with $A A F<0.1$ were removed.

The AAFs were summarized at each position, overall, and by group (ER+, ER-, control). Frequencies were also summarized for each gene and overall, across genes.

Gene-level analyses considered mutations in two ways: 1) as a continuous allele frequency, and 2) as a binary presence/absence of mutation variable. Gene-level analyses used logistic regression to compare the sum of continuous variant allele frequencies across variants in cases (all cases, as well as separately in ER + and ER-) versus controls. Additionally, gene-level analyses of the presence/absence of mutations across a gene were conducted with SKAT-0. In addition to the default weighting based on variant allele frequencies, secondary analyses also implemented a more stringent variant weighting where $\mathrm{C}->\mathrm{T}$ mutations were downweighted compared to other mutation types.

All models were adjusted for relevant covariates, including epithelial percentage, histologic impression, patient's age, year of biopsy (including a linear term for year, indicator for whether the biopsy was post-1992, year*1992 interaction due to an FFPE processing change), and SNP call rate. Sensitivity analyses were performed for the different variant QC criteria (strict, classical, and liberal). Primary analyses compared all cases (ER + and ER-) to controls, but secondary analyses considered pair-wise group comparisons. 
Analyses were also performed where genes were grouped into pre-defined gene-sets based on the annotation from the Qiagen candidate gene panel.

\section{Immunohistochemistry analysis}

In all BBD samples, expression of Ki67 and CD45 was assessed in up to 10 normal lobules using IHC. Immunostaining was performed using the following antibodies: CD45 (Abcam ab10559, 1:800), Ki67 (DAKO M7240, 1:100). Samples were deparaffinized with three changes of xylene, rehydrated in $95 \%$ ethanol and rinsed well in running distilled water. Slides were then placed in a preheated Antigen Retrieval solution $(\mathrm{pH}$ 6.0, DAKO) for $25 \mathrm{~min}$ and then cooled in the buffer for 25 minutes followed by a five-minute rinse in running distilled water. After the heat-inactivated epitope retrieval step, slides were placed on the DAKO Autostainer at room temperature for the following procedure. Sections were incubated with $3 \% \mathrm{H}_{2} \mathrm{O}_{2}$ for five minutes to inactivate the endogenous peroxides and then incubated in the primary antibody at dilutions listed above for 60 minutes at room temperature. Sections were rinsed with Tris-buffered saline/Triton-X100 (TBST) wash buffer and incubated with the secondary antibody (Envision (+) anti-mouse labeled polymer (HRP, K4001) for Ki67 and Envision (+) anti-rabbit labeled polymer (HRP, K4003) for CD45) for 30 minutes. Slides were then rinsed with TBST wash buffer and sections were incubated in 3,3'-diaminobenzidine (DAB+) (K3467, DAKO) for five minutes, counterstained with Gills I hematoxylin for one minute, followed by a three-minute tap water rinse to blue sections, dehydrated through graded alcohols and cleared in three changes of xylene and mounted with permanent mounting media. Staining was quantitated using Aperio ${ }^{\text {TM }}$ ImageScope Software (Leica Biosystems, Buffalo Grove, IL, USA). Ki67 was measured as percent positive nuclei and CD45 was measured as an $\mathrm{H}$ score (a combined measure of the intensity and extent of staining) [29-31]. CD45 and Ki67 were assessed as continuous measures. Associations across groups were evaluated as Wilcoxon rank-sum tests, and associations with mutational burden were evaluated with Spearman correlation.

\section{Results}

\section{Sample Characteristics}

The clinicopathologic characteristics of the samples selected for this study are shown in Table 1. Among the full BBD Cohort, a frequency-matched case-control sample set of BBD biopsies was selected based on outcome in follow-up at 16 years: incident ER+ BC (BBD-ER+), incident ER- BC (BBD-ER-) or cancer-free (BBDcontrol), matched on age at biopsy and year of biopsy or censoring. Selection criteria also included availability of blocks with adequate tissue for DNA extraction. Severity of BBD was the only feature that differed significantly among the three groups $(p=0.026)$. The BBD-controls included the highest percentage of non-proliferative disease $(n=25 ; 59.5 \%)$, while the BBD-ER- group had the highest proportion of proliferative disease without atypia $(n=19 ; 52.8 \%)$ and the BBD-ER+ group had the highest proportion of atypical hyperplasia $(n=9 ; 21.4 \%)$, consistent with previous studies [5].

\section{$\underline{\text { Gene-level associations }}$}


To address potentially artefactual FFPE variants, 12 combinations of variant filtering strategies and statistical analysis methods (classical, liberal and strict variant quality control (QC) filtering, combined with C-T weighted and un-weighted SKAT-O and logistic regression, described in Methods) were used to identify significant gene-level mutation burden differences between cancer (BBD-ER+ and BBD-ER-) and cancer-free (BBD-control) groups (Fig. 1A; Table 2). Full results are shown in Additional files 7-9. Through consensus analysis of association results shown as Fig. 1B, 10 genes (MED12, MSH2, BRIP1, PMS1, GATA3, MUC16, FAM175A, EXT2, MLH1 and TGFB1) of nominal significance $(\mathrm{p}<0.05)$ were found according to at least four methods.

After extensive sample- and variant-level quality control, common single nucleotide polymorphisms (SNP) variants detected in this cohort had highly consistent allele frequency distributions when compared with population frequencies derived from large-scale germline sequencing studies such as 1000 Genome Project and Exome Aggregation Consortium (ExAC), shown as Fig. 2A. The concordance of detected allele frequencies with population frequencies were persistent even when variants were stratified by nucleotide substitution type [See Additional File 10]. This strong concordance at the population-level suggests a solid basis for the association analyses. When comparing overall BBD cancer cases (BBD-ER+ and BBD-ER-) versus $\mathrm{BBD}$-controls, a volcano plot of gene-level association effect-sizes by the corresponding significance levels showed a skewed distribution, with more significant findings enriched for more mutations in cancerfree subjects (Fig. 2B). By further stratifying association analysis by type of BC (i.e. ER+ and ER-), the association differences were more profound when comparing BBD controls with BBD subjects with future ER- cancers than those with ER+ cancers, while the volcano plots remained skewed towards enrichment of protective associations [See Additional File 11].

\section{Mutational Signatures}

As we and others have shown, FFPE-derived sequencing may have distinct variant signatures collectively [15], and therefore de-novo mutational signature decomposition was conducted based on filtered variants for the entire BBD cohort, leading to four different mutational signatures shown in Fig. 3A: two of the observed signatures were primarily enriched for " $\mathrm{C}>\mathrm{T}$ " paraffin artifacts and highly similar to FFPE/chemistry signatures, which we previously identified in paired comparisons between matched frozen and FFPE samples [15]. One of the de-novo signatures (Signature-D) was found to be highly correlated with collection age of FFPE block (Fig. 3B and 3C). However, no statistically significant difference was found between this block-year associated signature with cancer status (Fig. 3D). Nonetheless, this highlights the necessity of strict global variant quality control measures beyond variant-level checks for FFPE sequencing data. Furthermore, we assessed a previously published BBD signature that was associated with risk of triple negative $B C$ for association in our dataset [13]; we did not observe presence of the signature in our sample, overall, or within ER+, ER-, or triple negative cases ( $p>0.05$, data not shown).

\section{$\underline{\text { Immunohistochemistry analysis }}$}

To follow-up on the findings that overall mutation was higher among BBD patients who remained cancerfree, we sought to investigate a potential hypothesis, where reduced mutational diversity is associated with 1) increased proliferation, or 2) reduced immune response. To investigate these hypotheses, we performed 
immunohistochemistry (IHC) analysis of Ki67 (as a marker of proliferation) and CD45 (as a marker of immune response) in normal lobules. Ki67 expression in normal lobules was very low, so analyses were not pursued further. However, CD45 expression was lower in BBD cases as compared to controls $(p=0.19)$, although not statistically significant, and was positively associated with mutational burden $(r=0.42$, $p=0.0031)$, most strongly in controls ( $r=0.50, p=0.005$; Fig. 4 A-D $)$.

\section{Germline mutation information}

Germline DNA was not available for the vast majority of subjects; however, a subset of 14 patients did have prior germline sequencing data available from other sources. Among these 14, 12 had no pathogenic mutations in pre-disposition genes. One BBD-ER- subject had a pathogenic mutation in BRCA1 that was also verified with clinical germline testing, and that $B R C A 1$ mutation was also detected in the BBD tissue in this study. One BBD-ER+ subject had a pathogenic mutation in $B L M$ but did not undergo confirmatory clinical germline testing, and the BLM mutation was not detected in the subject's BBD tissue in this study.

\section{Discussion}

In this study, 120 individuals diagnosed with BBD were studied to examine the association of somatic genetic variation with subsequent cancer development. The BBD controls with at least 16 years of cancerfree follow up were compared with BBD patients who developed ER+ or ER-invasive BC within 16 years, with comparison also between the two cancer groups. With a relatively balanced distribution of future cancer status, several genes were shown to have consistent associations with development of BC using 12 statistical methods, including SKAT-O and logistic regression in classical, liberal and strict variant QC schema. Skewed gene-level associations towards lower mutational burden were seen in the overall case population and in ER-cases, supporting the stratified analysis for studying BC risk. Our extensive variantlevel and mutational signature-based quality control assessments also highlight the challenges for analyzing biopsy-based BBD archival collections.

Other BBD sequencing-based studies have reported no significant mutations in original BBD tissues, and little to no overlap in the subsequent invasive $B C$ [11-13]. There are multiple possible reasons for the differences in results compared to our study. Each of these studies used surrogates of normal genomes to filter the data; in one study, tissue adjacent to the BBD lesion was used [11], which may inadequately account for field effects and lead to over-filtering. Rather, we used population germline frequencies from published SNP databases to validate our variant filtering and then compared the mutation spectrum between the three groups. Additionally, one prior study focused on a limited number of BBD cases with fibrocystic histology [12], while the other [11] included multiple BBD histological types. Soysal et al [12] reported that two "Tier 2" mutations (with features suggestive of fixation artefacts) in MAP3K1 and PIK3CA, were present in BBD as well as in the subsequent BC. They also reported three GATA3 mutations (one "likely genuine" and two Tier 2) that were found in tumors but not the fibrocystic lesions; we also identified GATA3 as a top gene in this study. A third study reported eight mutations shared between BBD lesions (with varied histologies) and subsequent invasive BC [13]. This manuscript also reported a de-novo mutational signature 
associated with future cancer risk; however, this was not reproduced in our study due possibly to the limited scope of our sequencing panel (93 genes v. whole-exome).

It is notable that mutations were more common in benign breast tissues of women who did not subsequently develop cancer. Many of the top 10 genes from our study are known to regulate cellular integrity, with half being involved in DNA repair (BRIP1, FAM175A), and particularly mismatch repair ( $M L H 1$, MSH2, PMS1). Regarding possible explanations for more common mutations in controls compared to cases, we hypothesize that cells with more DNA damage might induce an immune-mediated response that protects against cancer development. In support of this, it has been shown that CD45+ cells are present in normal breast tissue [16], are more abundant in BBD compared to normal breast tissues, and B cells are less frequent in BBD cases compared to controls[17]. In this study, CD45 expression was significantly higher with higher mutational burden and was somewhat lower in this series of BBD cases compared to controls, albeit not reaching statistical significance. There is a growing literature on somatic mutations in normal and benign tissues [18-20] leading to questions about the role that somatic mutation and clonal expansion may play in aging and disease, and it has been proffered that analyses of normal and precursor lesions are needed to understand how these may contribute, if at all, to cancer and other disease development [20]. Interestingly, these sequencing studies of normal tissues find mutations in many samples that do not appear to be destined for cancer, and varying hypotheses have been advanced including immune system involvement and tissue architecture [18]. Supporting an immune-mediated suppression of carcinogenesis, lower variant allele frequencies were noted in gene mutations with in-silico predicted neoantigens [18]. If such neoantigens result in mutant proteins, they may elicit an immune-mediated response; thus examining gene expression as well as the presence of immune mediator cells in tissue sections would be good next steps. Many of the significantly associated genes in our study are involved in DNA repair, with potential for recruiting an immune response to clear cells with DNA damage. Whether the increased mutational load results in changes more likely to be immunogenic in benign tissues is intriguing and is an interesting avenue for future research.

Strengths of this study include use of archival tissues from an annotated cohort with long follow-up for cancer outcomes, as well as careful quality control in study design, methods, and the analytical plan. Close attention was paid to handling of these data, including evaluating variant allele frequency differences depending on the nucleotide substitution, combined with multiple approaches to statistical analyses, and defining quality control criteria based on expected variation differences between matched FPPE and fresh frozen samples. In addition to defining top genes by statistical significance, we added additional rigor by requiring significance across multiple analytical approaches. Limitations include the use of older FFPE tissue which is more prone to sequencing artifacts; molecular preservation in such tissues often poses challenges for analysis, particularly for older samples when reagents and processing protocol were less standardized in clinical practice. However, while fresh or frozen tissue is more optimal for sequencing studies, noting the high concordance of single nucleotide variants (SNVs) with publicly available data lends confidence to our careful filtering approach. Furthermore, this study did not include germline sequencing data for filtering of potential somatic mutations in the benign tissue, nor paired sequencing with the subsequent tumor. Finally, due to a small sample size per group, power was limited to detect mutational differences, particularly for individual variants.

Page $11 / 23$ 
In summary, we identified genes associated with later cancer status, commonly involving DNA repair pathways, and interestingly the variant burden was significantly higher in controls than in either future ER+ or ER- cases. CD45 expression was associated with mutational burden, suggesting a possible role of immunosurveillance in impeding cancer development. Key gene-level findings warrant future validation in large cohort studies, and analyses in paired benign and subsequent tumor tissue.

\section{Abbreviations}

Benign breast disease (BBD)

Breast cancer $(B C)$

Deoxyribonucleic acid (DNA)

Formalin fixed, paraffin-embedded (FFPE)

Single nucleotide polymorphisms (SNP)

Variant allele frequency (VAF)

Estrogen receptor positive (ER+)

Estrogen receptor negative (ER-)

Quality control (QC)

Immunohistochemistry (IHC)

Single nucleotide variants (SNVs)

Molecular tag (MT)

False discovery rate (FDR)

Alternate allele frequency (AAF)

Non-negative matrix factorization (NMF)

Genome Aggregation Database (gnomAD)

Trans-Omics for Precision Medicine (TOPMED)

Immunohistochemistry (IHC)

Unique Molecular Tag (UMT)

QIAGEN GeneRead DNA FFPE Kit (QGR) 


\section{Declarations}

\section{Acknowledgements}

We thank study participants of the Mayo Clinic Benign Breast Disease Cohort for making this research possible, and Ann Westphal with assistance with manuscript preparation.

\section{Funding}

This work was supported by the National Cancer Institute via grant R01CA187112, and also by the Asante Foundation. These funding bodies had no role in the study design, data generation, data analysis and interpretation, nor manuscript writing.

Availability of data and materials

The datasets supporting the conclusions of this article are included within the article and its additional files.

Ethics approval and consent to participate

This work was approved by the Mayo Clinic Institutional Review Board (IRB \#75-87). As required by Mayo Clinic Institutional Review Board policies, all subject provided written research consent or authorization for use of their tissues and data.

Competing Interests

Authors have no competing interests to declare.

Consent for publication

Not applicable

\section{Authors' Contributions}

SJW and CW supervised statistical and bioinformatics analyses and interpretation, participated in study design, and drafting the manuscript. EPH and RAV participated in study design, statistical analyses, interpretation of results, and manuscript drafting. AVB and YL performed bioinformatics analyses, participated in interpretation of results, and manuscript drafting. SJM and MLS performed laboratory analysis of samples and participated in interpretation of results. MHF participated in study design and regulatory approvals. JMC1 participated in study design, review of tissue samples, and interpretation of results. MES participated in interpretation of results and manuscript drafting. DCR supervised laboratory analysis of samples, participated in study design, interpretation of results, and manuscript drafting. ACD supervised and coordinated project efforts; participated in study design, interpretation of results, and manuscript drafting. JMC2 supervised laboratory analysis of samples, and participated in study design, interpretation of results, and manuscript drafting. All authors read and approved the final manuscript. 


\section{References}

1. Antoniou AC, Easton DF: Models of genetic susceptibility to breast cancer. Oncogene 2006, 25(43):5898-5905.

2. Brody LC, Biesecker BB: Breast cancer susceptibility genes. BRCA1 and BRCA2. Medicine (Baltimore) 1998, 77(3):208-226.

3. Couch FJ, Hart SN, Sharma P, Toland AE, Wang X, Miron P, Olson JE, Godwin AK, Pankratz VS, Olswold C et al: Inherited mutations in 17 breast cancer susceptibility genes among a large triple-negative breast cancer cohort unselected for family history of breast cancer. J Clin Oncol 2015, 33(4):304-311.

4. American Cancer Society: Survival Rates for Breast Cancer In.

5. Hartmann LC, Sellers TA, Frost MH, Lingle WL, Degnim AC, Ghosh K, Vierkant RA, Maloney SD, Pankratz VS, Hillman DW et al: Benign breast disease and the risk of breast cancer. N Engl J Med 2005, 353(3):229-237.

6. Dupont WD, Page DL: Risk factors for breast cancer in women with proliferative breast disease. N Eng/ J Med 1985, 312(3):146-151.

7. Dupont WD, Parl FF, Hartmann WH, Brinton LA, Winfield AC, Worrell JA, Schuyler PA, Plummer WD: Breast cancer risk associated with proliferative breast disease and atypical hyperplasia. Cancer 1993, 71(4):1258-1265.

8. Darooei M, Khan F, Rehan M, Zubeda S, Jeyashanker E, Annapurna S, Shah A, Maddali S, Hasan Q: MED12 somatic mutations encompassing exon 2 associated with benign breast fibroadenomas and not breast carcinoma in Indian women. J Cell Biochem 2019, 120(1):182-191.

9. Danforth DN, Jr.: Genomic Changes in Normal Breast Tissue in Women at Normal Risk or at High Risk for Breast Cancer. Breast Cancer (Auckl) 2016, 10:109-146.

10. Danforth DN: Molecular profile of atypical hyperplasia of the breast. Breast Cancer Res Treat 2018, 167(1):9-29.

11. Rohan TE, Miller CA, Li T, Wang Y, Loudig O, Ginsberg M, Glass A, Mardis E: Somatic mutations in benign breast disease tissue and risk of subsequent invasive breast cancer. Br J Cancer 2018, 118(12):1662-1664.

12. Soysal SD, Ng CKY, Costa L, Weber WP, Paradiso V, Piscuoglio S, Muenst S: Genetic Alterations in Benign Breast Biopsies of Subsequent Breast Cancer Patients. Front Med (Lausanne) 2019, 6:166.

13. Zeng Z, Vo A, Li X, Shidfar A, Saldana P, Blanco L, Xuei X, Luo Y, Khan SA, Clare SE: Somatic genetic aberrations in benign breast disease and the risk of subsequent breast cancer. NPJ Breast Cancer 2020, 6:24.

14. Frank RD, Winham SJ, Vierkant RA, Frost MH, Radisky DC, Ghosh K, Brandt KR, Sherman ME, Visscher DW, Hartmann LC et al: Evaluation of 2 breast cancer risk models in a benign breast disease cohort. Cancer 2018, 124(16):3319-3328.

15. Bhagwate AV, Liu Y, Winham SJ, McDonough SJ, Stallings-Mann ML, Heinzen EP, Davila JI, Vierkant RA, Hoskin TL, Frost $M$ et al: Bioinformatics and DNA-extraction strategies to reliably detect genetic variants from FFPE breast tissue samples. BMC Genomics 2019, 20(1):689. 
16. Degnim AC, Brahmbhatt RD, Radisky DC, Hoskin TL, Stallings-Mann M, Laudenschlager M, Mansfield A, Frost $\mathrm{MH}$, Murphy L, Knutson $\mathrm{K}$ et al: Immune cell quantitation in normal breast tissue lobules with and without lobulitis. Breast Cancer Res Treat 2014, 144(3):539-549.

17. Degnim AC, Hoskin TL, Arshad M, Frost MH, Winham SJ, Brahmbhatt RA, Pena A, Carter JM, StallingsMann ML, Murphy LM et al: Alterations in the Immune Cell Composition in Premalignant Breast Tissue that Precede Breast Cancer Development. Clin Cancer Res 2017, 23(14):3945-3952.

18. Zhu M, Lu T, Jia Y, Luo X, Gopal P, Li L, Odewole M, Renteria V, Singal AG, Jang Y et al: Somatic Mutations Increase Hepatic Clonal Fitness and Regeneration in Chronic Liver Disease. Cell 2019, 177(3):608-621.e612.

19. Martincorena I: Somatic mutation and clonal expansions in human tissues. Genome Med 2019, 11(1):35.

20. Yizhak K, Aguet F, Kim J, Hess JM, Kübler K, Grimsby J, Frazer R, Zhang H, Haradhvala NJ, Rosebrock D et al: RNA sequence analysis reveals macroscopic somatic clonal expansion across normal tissues. Science 2019, 364(6444).

21. GeneGlobe [https://www.qiagen.com/us/geneglobe/]

22. Xu C, Nezami Ranjbar MR, Wu Z, DiCarlo J, Wang Y: Detecting very low allele fraction variants using targeted DNA sequencing and a novel molecular barcode-aware variant caller. BMC Genomics 2017, 18(1):5.

23. Kocher JP, Quest DJ, Duffy P, Meiners MA, Moore RM, Rider D, Hossain A, Hart SN, Dinu V: The Biological Reference Repository (BioR): a rapid and flexible system for genomics annotation. Bioinformatics 2014, 30(13):1920-1922.

24. Blokzijl F, Janssen R, van Boxtel R, Cuppen E: MutationalPatterns: comprehensive genome-wide analysis of mutational processes. Genome Med 2018, 10(1):33.

25. Tate JG, Bamford S, Jubb HC, Sondka Z, Beare DM, Bindal N, Boutselakis H, Cole CG, Creatore C, Dawson E et al: COSMIC: the Catalogue Of Somatic Mutations In Cancer. Nucleic Acids Res 2019, 47(D1):D941-d947.

26. Karczewski KJ, Francioli LC, Tiao G, Cummings BB, Alföldi J, Wang Q, Collins RL, Laricchia KM, Ganna A, Birnbaum DP et al: The mutational constraint spectrum quantified from variation in 141,456 humans. Nature 2020, 581(7809):434-443.

27. NHLBI TOPMed Program: Sequencing of 53,831 diverse genomes from the NHLBI TOPMed Program. In.; 2019.

28. Münz M, Ruark E, Renwick A, Ramsay E, Clarke M, Mahamdallie S, Cloke V, Seal S, Strydom A, Lunter G et al: CSN and CAVA: variant annotation tools for rapid, robust next-generation sequencing analysis in the clinical setting. Genome Med 2015, 7(1):76.

29. Hirsch FR, Varella-Garcia M, Bunn PA, Jr., Di Maria MV, Veve R, Bremmes RM, Barón AE, Zeng C, Franklin WA: Epidermal growth factor receptor in non-small-cell lung carcinomas: correlation between gene copy number and protein expression and impact on prognosis. J Clin Oncol 2003, 21(20):3798-3807.

30. John T, Liu G, Tsao MS: Overview of molecular testing in non-small-cell lung cancer: mutational analysis, gene copy number, protein expression and other biomarkers of EGFR for the prediction of 
response to tyrosine kinase inhibitors. Oncogene 2009, 28(1):S14-S23.

31. Calculating H-Score [https://ascopost.com/issues/april-10-2015/calculating-h-score/]

\section{Tables}

Table 1. Cohort characteristics

\begin{tabular}{|c|c|c|c|c|c|}
\hline & $\begin{array}{l}\text { Cancer Free at } 16 \\
\text { years } \\
(\mathrm{N}=42)\end{array}$ & $\begin{array}{l}\text { ER Negative } \\
B C \\
(\mathrm{~N}=36)\end{array}$ & $\begin{array}{l}\text { ER Positive } \\
\text { BC } \\
(\mathrm{N}=42)\end{array}$ & $\begin{array}{l}\text { Total } \\
(\mathrm{N}=120)\end{array}$ & $\begin{array}{l}\mathrm{P} \\
\text { Value }\end{array}$ \\
\hline Age & & & & & 0.416 \\
\hline$<45$ & $11(26.2 \%)$ & $11(30.6 \%)$ & $7(16.7 \%)$ & $\begin{array}{l}29 \\
(24.2 \%)\end{array}$ & \\
\hline $45-55$ & $19(45.2 \%)$ & $14(38.9 \%)$ & $16(38.1 \%)$ & $\begin{array}{l}49 \\
(40.8 \%)\end{array}$ & \\
\hline$>55$ & $12(28.6 \%)$ & $11(30.6 \%)$ & $19(45.2 \%)$ & $\begin{array}{l}42 \\
(35.0 \%)\end{array}$ & \\
\hline Histologic Impression & & & & & 0.026 \\
\hline Non-Proliferative Disease & $25(59.5 \%)$ & $14(38.9 \%)$ & $19(45.2 \%)$ & $\begin{array}{l}58 \\
(48.3 \%)\end{array}$ & \\
\hline $\begin{array}{l}\text { Proliferative Disease } \\
\text { Without Atypia }\end{array}$ & $16(38.1 \%)$ & $19(52.8 \%)$ & $14(33.3 \%)$ & $\begin{array}{l}49 \\
(40.8 \%)\end{array}$ & \\
\hline Atypical Hyperplasia & $1(2.4 \%)$ & $3(8.3 \%)$ & $9(21.4 \%)$ & $\begin{array}{l}13 \\
(10.8 \%)\end{array}$ & \\
\hline Atrophy & & & & & 0.086 \\
\hline N-Miss & 2 & 3 & 3 & 8 & \\
\hline None & $9(22.5 \%)$ & $13(39.4 \%)$ & $6(15.4 \%)$ & $\begin{array}{l}28 \\
(25.0 \%)\end{array}$ & \\
\hline Partial & $16(40.0 \%)$ & $13(39.4 \%)$ & $23(59.0 \%)$ & $\begin{array}{l}52 \\
(46.4 \%)\end{array}$ & \\
\hline Complete & $15(37.5 \%)$ & $7(21.2 \%)$ & $10(25.6 \%)$ & $\begin{array}{l}32 \\
(28.6 \%)\end{array}$ & \\
\hline Year of BBD & & & & & 0.452 \\
\hline Mean (SD) & $1986(8)$ & 1987 (8) & $1988(9)$ & $1987(8)$ & \\
\hline Range & $1969-1996$ & $1970-1999$ & $\begin{array}{l}1972- \\
2001\end{array}$ & $\begin{array}{l}1969- \\
2001\end{array}$ & \\
\hline
\end{tabular}


Table 2. Association results of leading genes (top 10 genes, with $P<0.05$ in 4 out of 12 methods) 


\begin{tabular}{|c|c|c|c|c|c|c|c|}
\hline Gene & $\begin{array}{l}\text { SKAT-O } \\
\text { p }\end{array}$ & $\begin{array}{l}\text { SKAT-O } \\
\text { p, } \\
\text { weighted }\end{array}$ & $\begin{array}{l}\text { Logistic } \\
\text { Regression } \\
\text { OR }\end{array}$ & $\begin{array}{l}\text { Logistic } \\
\text { Regression } \\
\mathrm{p}\end{array}$ & $\begin{array}{l}\text { Logistic } \\
\text { Regression } \\
\text { OR, } \\
\text { weighted }\end{array}$ & $\begin{array}{l}\text { Logistic } \\
\text { Regression } \\
p_{\text {, }} \\
\text { weighted }\end{array}$ & Analysis \\
\hline \multirow[t]{3}{*}{ MED12 } & $0.0475^{\star}$ & $0.0390 *$ & $\begin{array}{l}0.9239 \\
(0.8455 \\
0.9916)\end{array}$ & $0.0268^{*}$ & $\begin{array}{l}0.8138 \\
(0.66, \\
0.9681)\end{array}$ & 0.0189* & classic \\
\hline & $0.0067 * \star$ & 0.0016 ** & $\begin{array}{l}0.9472 \\
(0.8917 \\
0.9941)\end{array}$ & $0.0261^{*}$ & $\begin{array}{l}0.8441 \\
(0.7129 \\
0.9696)\end{array}$ & $0.0148 *$ & liberal \\
\hline & $0.0445^{\star}$ & $0.0342^{*}$ & $\begin{array}{l}0.9205 \\
(0.8368 \\
0.9923)\end{array}$ & $0.0293^{*}$ & $\begin{array}{l}0.8326 \\
(0.686 \\
0.9721)\end{array}$ & $0.0189 *$ & strict \\
\hline \multirow[t]{3}{*}{ MSH2 } & $0.0203^{*}$ & $0.0445^{\star}$ & $\begin{array}{l}0.7539 \\
(0.5546 \\
0.9418)\end{array}$ & $0.0078 * \star$ & $\begin{array}{l}0.4294 \\
(0.1775 \\
0.8518)\end{array}$ & $0.0085^{\star \star}$ & classic \\
\hline & 0.0544 & 0.0856 & $\begin{array}{l}0.8651 \\
(0.7189 \\
0.9902)\end{array}$ & $0.0339 *$ & $\begin{array}{l}0.618 \\
(0.3305, \\
0.9584)\end{array}$ & $0.0277^{\star}$ & liberal \\
\hline & $0.0284^{*}$ & $0.0145^{\star}$ & $\begin{array}{l}0.6938 \\
(0.4566 \\
0.9331)\end{array}$ & $0.0097 * \star$ & $\begin{array}{l}0.3202 \\
(0.1025, \\
0.7632)\end{array}$ & $0.0043^{\star *}$ & strict \\
\hline \multirow[t]{3}{*}{ BRIP1 } & $0.0252^{\star}$ & 0.1015 & $\begin{array}{l}0.8686 \\
(0.7356 \\
0.9874)\end{array}$ & $0.0301 *$ & $\begin{array}{l}0.7081 \\
(0.4852 \\
0.9707)\end{array}$ & $0.0313^{*}$ & classic \\
\hline & $0.0087 * \star$ & $0.0319 *$ & $\begin{array}{l}0.8873 \\
(0.7758 \\
0.9777)\end{array}$ & $0.0131 *$ & $\begin{array}{l}0.7271 \\
(0.5182, \\
0.9436)\end{array}$ & $0.0146^{*}$ & liberal \\
\hline & $0.0468 *$ & 0.0809 & $\begin{array}{l}0.864 \\
(0.7049 \\
1.002)\end{array}$ & 0.0542 & $\begin{array}{l}0.7046 \\
(0.4418 \\
0.9956)\end{array}$ & $0.0469 *$ & strict \\
\hline \multirow[t]{3}{*}{ PMS1 } & 0.0519 & 0.0935 & $\begin{array}{l}0.8117 \\
(0.6799 \\
0.949)\end{array}$ & $0.0081^{* *}$ & $\begin{array}{l}0.6126 \\
(0.4005 \\
0.8929)\end{array}$ & $0.0101^{\star}$ & classic \\
\hline & 0.0684 & 0.0842 & $\begin{array}{l}0.8595 \\
(0.745 \\
0.9704)\end{array}$ & $0.0134^{\star}$ & $\begin{array}{l}0.6628 \\
(0.4569 \\
0.9149)\end{array}$ & $0.0116^{\star}$ & liberal \\
\hline & $0.0165^{\star}$ & $0.0136 *$ & $\begin{array}{l}0.7846 \\
(0.6447 \\
0.9309)\end{array}$ & $0.0047 * \star$ & $\begin{array}{l}0.6053 \\
(0.3996 \\
0.865)\end{array}$ & $0.0049 * \star$ & strict \\
\hline \multirow[t]{2}{*}{ GATA3 } & $0.0046 * \star$ & 0.2446 & $\begin{array}{l}0.7949 \\
(0.5809 \\
1.004)\end{array}$ & 0.0544 & $\begin{array}{l}0.4899 \\
(0.2246 \\
0.938)\end{array}$ & $0.0304 *$ & classic \\
\hline & $0.0130 *$ & 0.4957 & $\begin{array}{l}0.8798 \\
(0.7154, \\
1.043)\end{array}$ & 0.1439 & $\begin{array}{l}0.6355 \\
(0.3493 \\
1.059)\end{array}$ & 0.0832 & liberal \\
\hline
\end{tabular}




\begin{tabular}{|c|c|c|c|c|c|c|c|}
\hline & $0.0051^{* *}$ & $0.0175^{\star}$ & $\begin{array}{l}0.8086 \\
(0.5848 \\
1.028)\end{array}$ & 0.0861 & $\begin{array}{l}0.4766 \\
(0.2047 \\
0.912)\end{array}$ & $0.0233^{*}$ & strict \\
\hline \multirow[t]{3}{*}{ MUC16 } & $0.0151 *$ & $0.0021^{* *}$ & $\begin{array}{l}0.9875 \\
(0.9726 \\
0.999)\end{array}$ & $0.0327 *$ & $\begin{array}{l}0.9588 \\
(0.9153 \\
0.9927)\end{array}$ & $0.0157 *$ & classic \\
\hline & 0.1559 & 0.1089 & $\begin{array}{l}0.9934 \\
(0.9847 \\
1.001)\end{array}$ & 0.0746 & $\begin{array}{l}0.9767 \\
(0.9494 \\
0.9992)\end{array}$ & $0.0424^{*}$ & liberal \\
\hline & 0.1247 & 0.0875 & $\begin{array}{l}0.988 \\
(0.9723,1)\end{array}$ & 0.0586 & $\begin{array}{l}0.9664 \\
(0.9259 \\
0.9982)\end{array}$ & $0.0376^{*}$ & strict \\
\hline \multirow[t]{3}{*}{ EXT2 } & 0.1100 & 0.1604 & $\begin{array}{l}0.8305 \\
(0.6589 \\
0.997)\end{array}$ & $0.0461^{*}$ & $\begin{array}{l}0.5994 \\
(0.3212 \\
1.01)\end{array}$ & 0.0549 & classic \\
\hline & $0.0404^{*}$ & $0.0494^{*}$ & $\begin{array}{l}0.8593 \\
(0.72, \\
0.9822)\end{array}$ & $0.0244^{*}$ & $\begin{array}{l}0.6348 \\
(0.3789 \\
0.9543)\end{array}$ & $0.0277 *$ & liberal \\
\hline & 0.1435 & 0.1836 & $\begin{array}{l}0.8292 \\
(0.6447 \\
1.009)\end{array}$ & 0.0620 & $\begin{array}{l}0.6434 \\
(0.3599 \\
1.038)\end{array}$ & 0.0718 & strict \\
\hline \multirow[t]{3}{*}{ FAM175A } & 0.0657 & 0.1724 & $\begin{array}{l}0.7249 \\
(0.4568 \\
1.014)\end{array}$ & 0.0612 & $\begin{array}{l}0.3819 \\
(0.09252, \\
1.061)\end{array}$ & 0.0669 & classic \\
\hline & $0.0103^{*}$ & $0.0181^{*}$ & $\begin{array}{l}0.7172 \\
(0.5108 \\
0.9316)\end{array}$ & $0.0095^{\star \star}$ & $\begin{array}{l}0.3513 \\
(0.1179 \\
0.8153)\end{array}$ & $0.0114^{*}$ & liberal \\
\hline & 0.1628 & 0.1461 & $\begin{array}{l}0.7465 \\
(0.4696 \\
1.05)\end{array}$ & 0.0981 & $\begin{array}{l}0.4276 \\
(0.1126 \\
1.089)\end{array}$ & 0.0793 & strict \\
\hline \multirow[t]{3}{*}{ MLH1 } & 0.1012 & 0.0885 & $\begin{array}{l}0.8551 \\
(0.7103 \\
1.005)\end{array}$ & 0.0573 & $\begin{array}{l}0.6972 \\
(0.4748 \\
0.9608)\end{array}$ & $0.0267 *$ & classic \\
\hline & 0.1320 & 0.0929 & $\begin{array}{l}0.8819 \\
(0.7573 \\
1.002)\end{array}$ & 0.0529 & $\begin{array}{l}0.7201 \\
(0.5073 \\
0.9604)\end{array}$ & $0.0244 *$ & liberal \\
\hline & 0.0895 & $0.0168^{*}$ & $\begin{array}{l}0.8393 \\
(0.672, \\
1.021)\end{array}$ & 0.0801 & $\begin{array}{l}0.7335 \\
(0.511 \\
0.9976)\end{array}$ & $0.0482^{*}$ & strict \\
\hline \multirow[t]{2}{*}{ TGFB1 } & 0.1689 & 0.1067 & $\begin{array}{l}0.7878 \\
(0.542, \\
1.077)\end{array}$ & 0.1381 & $\begin{array}{l}0.5017 \\
(0.1886 \\
1.029)\end{array}$ & 0.0610 & classic \\
\hline & $0.0070 * *$ & $0.0146^{*}$ & $\begin{array}{l}0.7198 \\
(0.5139 \\
0.9364)\end{array}$ & $0.0118 *$ & $\begin{array}{l}0.4107 \\
(0.1599 \\
0.8326)\end{array}$ & $0.0097 * \star$ & liberal \\
\hline
\end{tabular}




\begin{tabular}{|c|c|c|c|c|c|}
\hline 0.6571 & 0.6260 & $\begin{array}{l}0.8737 \\
(0.5926, \\
1.236)\end{array}$ & 0.4487 & $\begin{array}{l}0.6909 \\
(0.2797, \\
1.412)\end{array}$ & 0.3246 \\
\hline
\end{tabular}

$p$-values less than two thresholds: $p<0.05\left(^{*}\right), p<0.01\left(^{* *}\right)$

\section{Figures}

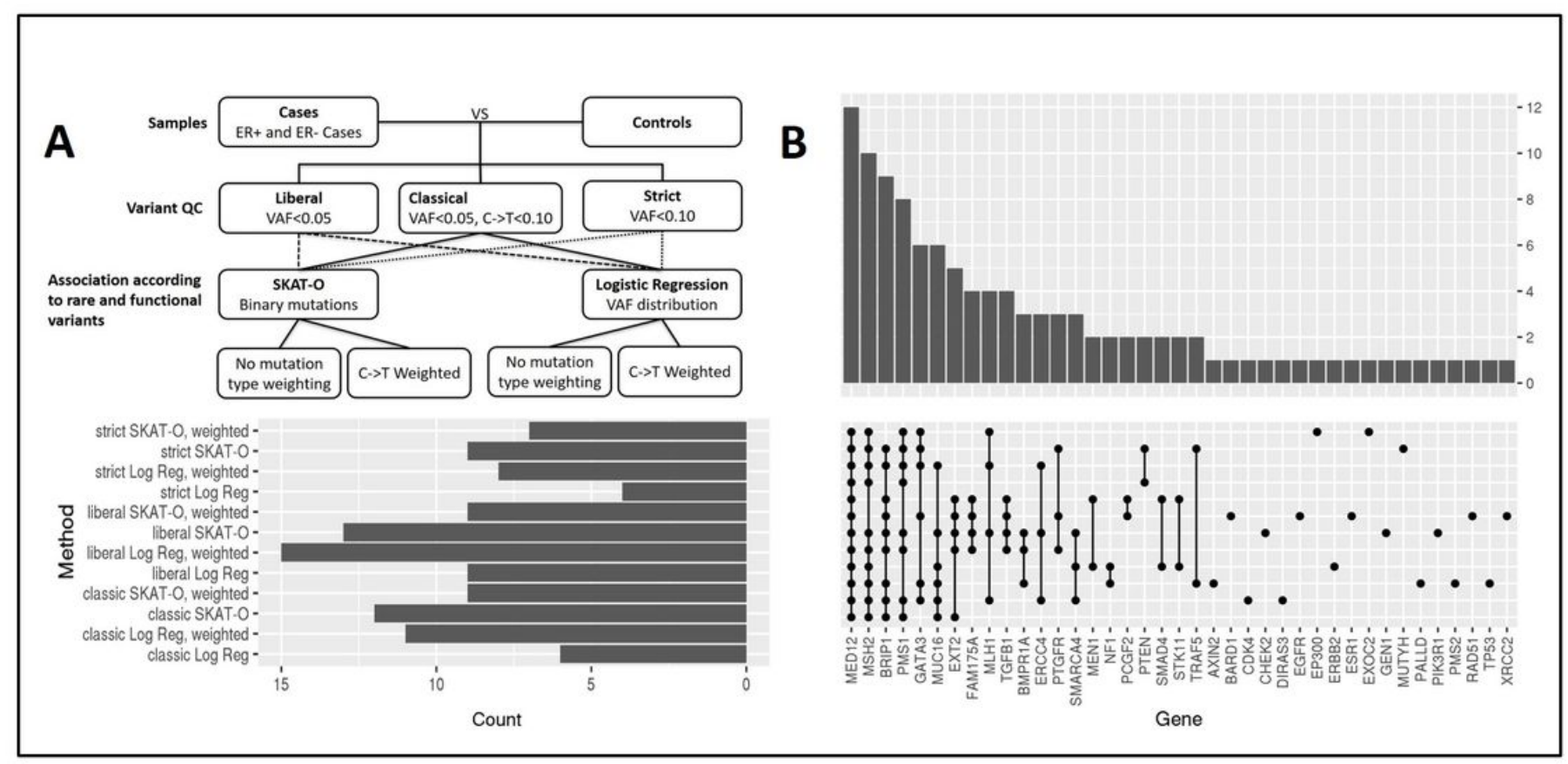

\section{Figure 1}

Gene-level association findings between cases (BBD with future cancer events) and controls (BBD without future cancer events up to 16 years): (A) analytical flows of 12 association methods. (B) Histogram with connected dot-plot summarizing consensus of significant genes detected by 12 methods.
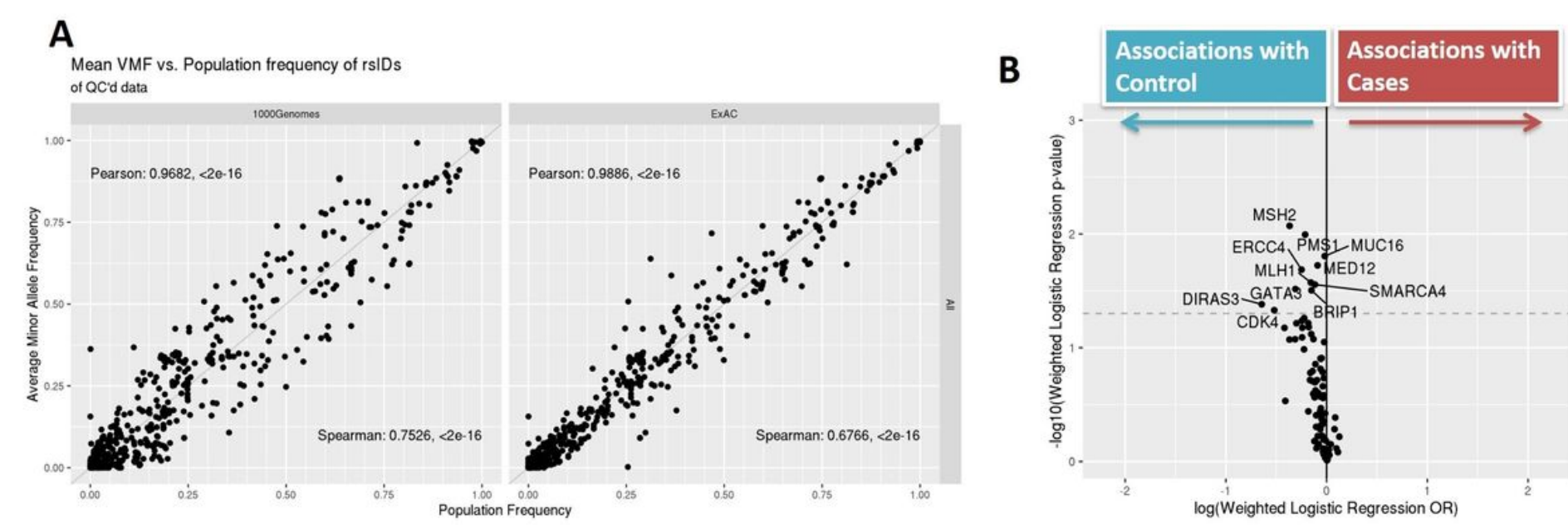


\section{Figure 2}

Variant concordances with normal genetics finding and gene-level volcano plots: (A) population frequency's variant-level ( $x$-axis) concordances with observed allele frequencies in this BBD cohort ( $y$-axis). Volcano plots of logistic regression-based odds-ratio (OR) and statistical significance, for all the cases v. controls (B).

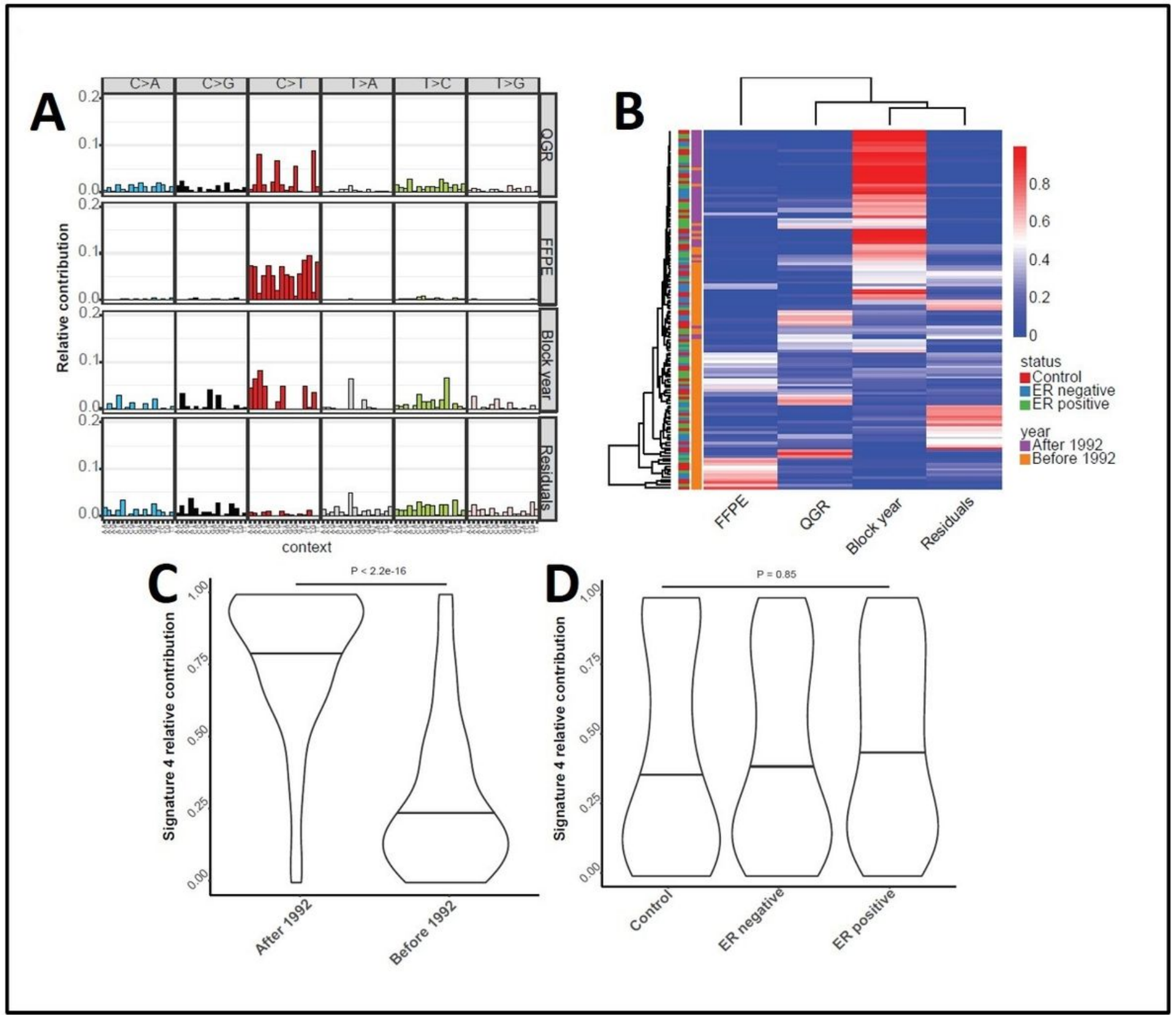

\section{Figure 3}

De-novo mutational signatures of entire dataset: (A) four dinucleotide signatures found through NMF. (B) heatmap of found de-novo signatures' coefficients across all samples. (C) Violin plots of signature-D's coefficients with respect to block-year (after v. before 1992). (D) Violin plots of signature-D's coefficients with respect to sample groups (control, ER-negative, and ER-positive). 


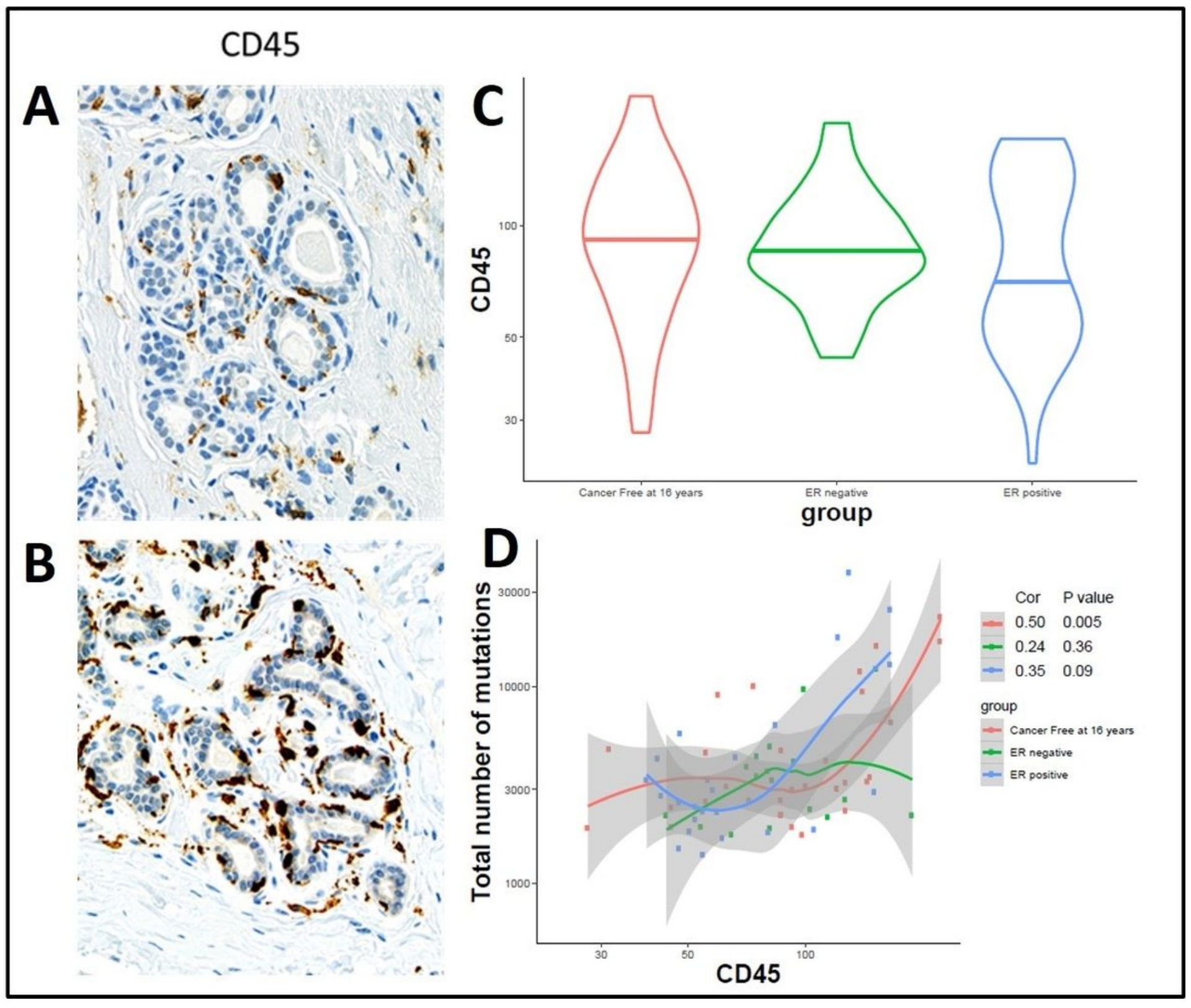

Figure 4

Expression of CD45 by group and mutational burden. $\mathrm{CD} 45$ is presented as an $\mathrm{H}$-score. A) Example staining of low CD 45 (18.62). B) Example staining of high CD45 (60.15). C) CD45 H score by group. D) CD 45 H score by mutational burden.

\section{Supplementary Files}

This is a list of supplementary files associated with this preprint. Click to download.

- SupplementaryFigure1.jpg

- SupplementaryFigure2.jpg

- SupplementaryFigure3.jpg

- SupplementaryFigure4.jpg 
- SupplementaryFigure5.jpg

- SupplementaryFigure6.jpg

- TableS1metadatadeidentified.xlsx

- TableS2mutationalsignatures.xlsx

- Tables3resultscasecontrol.xIsx

- Tables4resultserp.xIsx

- TableS5resultsern.xlsx 